\title{
Impact of channel errors and data aggregation on throughput in $\mathrm{THz}$ communications
}

\author{
Noureddine Boujnah, Saim Ghafoor and Alan Davy \\ Waterford Institute of Technology \\ TSSG, Waterford, Ireland \\ Email: bnoureddine@tssg.org, sghafoor@tssg.org, adavy@tssg.org
}

\begin{abstract}
In Terahertz communications, the throughput can be affected by incoming packet rate from upper layers and channel bit error probability. The techniques like block aggregation and retransmission can be used to mitigate these effects. The impact of data aggregation and forward error control on the overall throughput of Terahertz communications is shown and modeled in this paper. The throughput is also analyzed based on buffer status. The results suggest that using block aggregation with forwarding error control can enhance the useful throughput and reduce the buffer load.
\end{abstract}

\section{INTRODUCTION}

Nowadays, ultra-high data rate transmission is possible using Terahertz (THz) gap [1], the frequency band ranging from $0.1 T H z$ to $10 T H z$ offers tens of available $\mathrm{GHz}$ windows characterized by low attenuation. Although its potential, $\mathrm{THz}$ transmission is still limited by the distance, multipath propagation and affected by atmospheric conditions. Most of the work in $\mathrm{THz}$ communication domain focused on antenna, device and channel characterization, very less effort has been seen on $\mathrm{THz}$ communication protocols [3], [4], their functionalities and impact on the overall throughput. Therefore, in this paper, the impact of different communication layer functionalities like data aggregation, buffering, and frame error control (FEC), on the overall throughput of the $\mathrm{THz}$ system is shown and modeled. Different techniques are used in different combinations to analyze the throughput. Initially, it is shown that using block aggregation and FEC can enhance system throughput and also the data waiting for transmission in the buffer.

The paper is organized as follows. In Section II, we describe the communication layer functionalities, which can affect the throughput. In Section IV, we show our simulation results of throughput and buffer status. Finally, the conclusion is presented in Section V.

\section{FRAME AND ERROR MODELING}

\section{A. Packet to frame conversion}

For high data rate traffic system, packet size can exceed 10000 bytes, then, packets should be converted into frames to adapt with the wireless link. Let $L_{p}(i)$ be the size of an arriving packet $i, N_{p}(t)$ the number of packets coming between 0 and $t$, and $N_{\text {frames }}(t)$ the number of generated frames during the same period:

$$
N_{\text {frame }}(t)\left(L_{f}-L_{0}\right)=\sum_{i=1}^{N_{p}(t)} L_{p}(i)
$$

$L_{f}$ is the frame size and $L_{0}$ is the frame overhead. And the frame arrival rate:

$$
\lambda_{\text {frame }}=\frac{1}{\left(L_{\text {frame }}-L_{0}\right)} \lim _{t \rightarrow \infty}\left[\frac{N_{p}(t)}{t} \frac{1}{N_{p}(t)} \sum_{i=1}^{N_{p}(t)} L_{p}(i)\right]
$$

The frame arrival rate is:

$$
\lambda_{\text {frame }}=\frac{\bar{L}_{p}}{L_{\text {frame }}-L_{0}} \lambda_{p}
$$

Packets are split into blocks, to each is attached a CRC field and preamble. A frame is then built using blocks aggregation.

\section{B. Frame transmission time}

One time slot $T$ includes the time to transmit a frame, receive a reply, and propagation/processing delays.

$$
T=T_{\text {syn }}+2 \frac{D_{\max }}{c}+\frac{L_{\text {frame }}}{R}+\frac{L_{\text {ack }}}{R_{A C K}}+T_{g}
$$

Where, $R$ is the bit rate and is assumed to be 100 Gbps, $D_{\max }$ is the maximum distance between TX and RX, $T_{\text {syn }}$ is the synchronization time between TX and RX, $L_{A C K}$ is the length of acknowledgment frame from the receiver and $T_{g}$ the guard period including processing time at transmitter and the receiver, where $T$ depends on transceiver technology and system requirement. 


\section{Error control}

Frame error rate (FER) depends on frame length and bit error probability. Let $P_{b}$ the bit error probability(BER), the frame error rate is:

$$
P_{\text {frame }}=1-\left(1-P_{b}\right)^{L_{\text {frame }}}
$$

and the average number of frame re-transmission is:

$$
\bar{L}_{\text {frame }}=\frac{1}{1-P_{\text {frame }}}
$$

Cyclic Redundancy check (CRC) at frame level and FEC before modulation procedure are used to enhance the link quality by increasing error detection and correction capabilities respectively.

\section{ThroughPUT ANALYSIS}

The useful throughput with retransmission and without blocks aggregation, is given by:

$$
\bar{R}=\frac{L_{\text {frame }}}{T} \sum_{1}^{+\infty} \frac{P(l)}{l}=\frac{L_{\text {frame }}}{T} \frac{\log \left(\bar{L}_{\text {frame }}\right)}{\bar{L}_{\text {frame }}-1}
$$

While, the average throughput with block aggregation is:

$$
\bar{R}_{b}=\frac{L_{\text {frame }}}{T} \sum_{1}^{+\infty} \frac{P(l)}{l}=\frac{L_{\text {frame }}}{T} \frac{\log \left(\bar{L}_{b}\right)}{\bar{L}_{b}-1}
$$

Where $\bar{L}_{b}$ and $P(l)$ are the mean number of blocks retransmissions and probability of frame success after $l-1$ transmission failures respectively. the arrival rate of frames coming to the TX buffer is given by:

$$
\lambda_{\text {buffer }}=\lambda_{\text {frame }}+\frac{P_{\text {frame }}}{T}
$$

\section{Simulations AND PERFORMANCE}

In figure 1, we plot the useful throughput as function of BER for a frame size equal to $L_{\text {frame }}=10000$ bits and transmission time slot $T=10^{-6} \mathrm{~s}$. Using both blocks aggregation and FEC enhances the useful throughput.

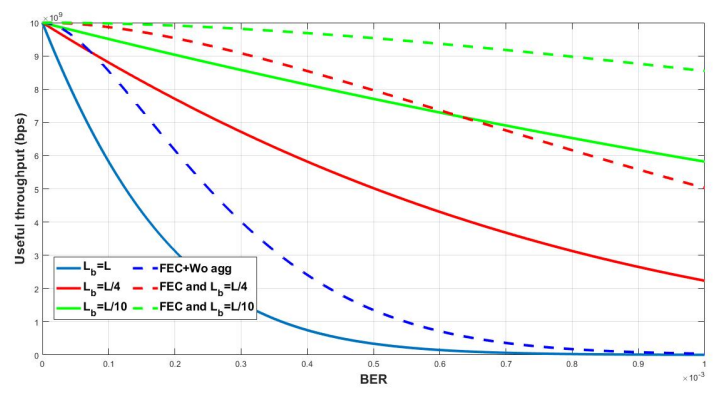

Fig. 1. Useful throughput for different configurations
Figure 2 shows the evolution of data at the transmitter buffer waiting for transmission and the average for different configuration, frames are generated according to Eq.1 and Eq.2, packets arrival follows a Poisson distribution with parameter $\lambda_{p}$. We remark that using block aggregation method outperforms the technique when no aggregation is used. The bit error rate is $B E R=10^{-6}$, the bit rate is $R=100 G b p s$ and the simulation time is $T_{s}=1 s$.

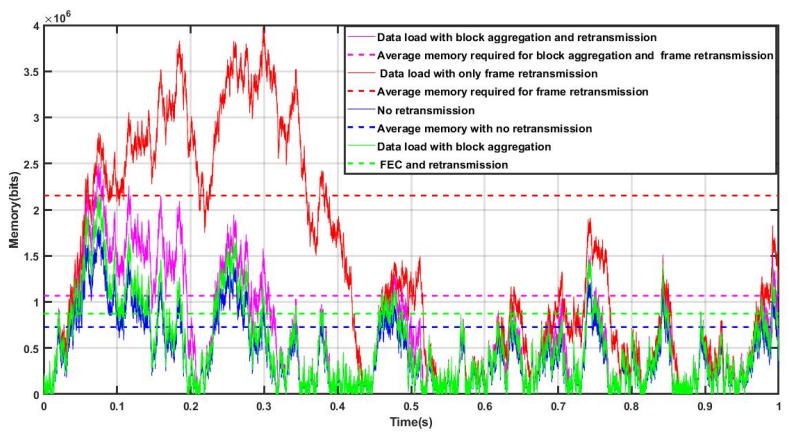

Fig. 2. Evolution of the amount of data in TX buffer for different network configurations

\section{Conclusion}

In this paper, we have shown that it is possible to increase transmission throughput for $\mathrm{THz}$ system using packet segmentation and block aggregation. Block aggregation with retransmission can enhance considerably the system performance by increasing link throughput and reducing the amount of data waiting at the buffer. As a perspective, we will focus on the optimal frame and block size.

\section{ACKNOWLEDGMENT}

This work has received funding from the European Union's Horizon 2020 research and innovation programme under grant agreement No 761579 (TERAPOD)

\section{REFERENCES}

[1] Chinni, V. K., Zezaoui, M., Coinon, C., Wallart, X., Pevtavit, E., Larnpin, J. F., Ducournau, G. (2018). Indoor 100 Gbit/s THz data link in the $300 \mathrm{GHz}$ band using fast photodiodes. 2018 25th International Conference on Telecommunications (ICT). doi:10.1109/ict.2018.8464945

[2] J. Jornet and I. Akyildiz, Channel Modeling and Capacity Analysis for Electromagnetic Wireless Nanonetworks in the Terahertz Band, IEEE Transactions on Wireless Communications, vol. 10, no. 10, pp. 3211 3221, 2011.

[3] X.W.Yao, J.Jornet TAB-MAC: Assisted beamforming MAC protocol for Terahertz communication networks Nano communication Network(Elsiever) Journal, vol. 9, pp.36-42,2016

[4] C. Han, W.Tong, X Wu A memory-assisted MAC protocol with angular-division-multiplexing in terahertz networks IEEE International Conference on Communication(ICC), May 2017, 